\title{
Bilateral nephromegaly and arthritis: A rare presentation of acute lymphoblastic leukemia*
}

\author{
Tapas Kumar Sabui ${ }^{1 \#}$, Syamal Sardar ${ }^{2}$, Sumanta Laha ${ }^{3}$, Abhishek Roy ${ }^{4}$ \\ ${ }^{1}$ Department of Pediatrics \& Neonatology, Institute of Post Graduate Medical Education \& Research, Kolkata, India \\ ${ }^{2}$ Department of Neonatology, Institute of Post Graduate Medical Education \& Research, Kolkata, India \\ ${ }^{3}$ Department of Pediatrics, Burdwan Medical College \& Hospital, Burdwan, India \\ ${ }^{4}$ Department of Pediatrics, North Bengal Medical College \& Hospital, Darjeeling, India \\ Email: "\#sabui@gmail.com
}

Received 30 May 2013; revised 28 June 2013; accepted 5 July 2013

Copyright (C 2013 Tapas Kumar Sabui et al. This is an open access article distributed under the Creative Commons Attribution License, which permits unrestricted use, distribution, and reproduction in any medium, provided the original work is properly cited.

\begin{abstract}
A 2.5-year-old boy presented with fever, intermittent small joint arthritis of hands and feet, bilateral nephromegaly with normal hemogram and uric acid level. Bone marrow aspiration revealed pre-B acute lymphoblastic leukemia without leukemic infiltration of kidneys. Leukemia should be suspected in any patient with arthritis and nephromegaly.
\end{abstract}

Keywords: Acute Lymphoblastic Leukemia; Nephromegaly; Arthritis

\section{INTRODUCTION}

Acute lymphoblastic leukemia (ALL) is a common disease and occasionally presents with rare features. However, bilateral kidney enlargement and intermittent small joint arthritis is extremely rare and this combination has never been described in literature till date. Hereby we report such a case with small joint arthritis and nephromegaly as an initial manifestation of ALL.

\section{CASE REPORT}

A 2.5-year-old male child born out of non consanguineous marriage was referred to our Pediatric Rheumatology clinic for opinion with history of fever for a short duration and intermittent arthritis of small joints of both hands and feet. He had two similar episodes of arthritis of hands, feet and ankle joint in last 3 months. It was treated by local pediatrician and he responded well to analgesics. He was clinically asymptomatic in between these acute illnesses.

His anthropometric measurements were within normal

${ }^{*}$ Conflict of interest: Nil.

" Corresponding author. limits. He was febrile and his interphalangeal, metacarpo-phalangeal joints of hands (Figure 1) and small joints of feet were tender and inflammed. Abdominal examination revealed bilateral palpable renal masses (left more than right). There was no pallor, hepatosplenomegaly or muco-cutaneous haemorrhagic spots. The child was normotensive and examination of others systems were within normal limit.

Complete hemogram showed hemoglobin of 14.6 $\mathrm{gm} / \mathrm{dl}$, total leucocyte count of $8400 / \mathrm{mm}^{3}$, neutrophil $48 \%$, lymphocyte $50 \%$, eosinophil $2 \%$, platelet count 2.4 lacs $/ \mathrm{mm}^{3}$ and normocytic normochromic RBC. His ESR and ASO titer were $28 \mathrm{~mm}$ in $1^{\text {st }}$ hour and $400 \mathrm{IU} / \mathrm{ml}$ respectively. Biochemical parameters including uric acid level, renal function and hepatic function tests were within normal limits. An increased uric acid value of $13.5 \mathrm{mg} / \mathrm{dl}$ was documented later during hospital stay. $\mathrm{X}$-ray of both hands and feet were normal. Bilaterally enlarged kidneys were documented with ultrasonography of abdomen. The size of right and left kidney was 11.2 $\mathrm{cm} \times 5.5 \mathrm{~cm}$ and $12 \mathrm{~cm} \times 6 \mathrm{~cm}$ respectively. There was loss of cortico-medullary differentiation. CT abdomen revealed diffusely enlarged and bulky kidneys with delayed contrast excretion and no focal mass lesion (Figure 2). Kidney biopsy showed hyperplasia and hypertrophy of parenchymal cells with infiltration of the interstitium by round cells with normochromatic nuclei. There was no definite evidence of leukemic infiltration. Bone marrow examination revealed markedly hypercellular marrow packed with blast cells which were cytomorphologically lymphoblasts. The blast cells were Periodic Acid Schiff (PAS) positive but negative for Sudan Black and Myeloperoxidase (MPO). On flow cytometric analysis, the blast cells expressed cCD79a, CD19 and CD10. CD19/CD10 co-expression was present in 95\% of these cells. CD20, CD34, HLADR, Kappa and Lambda 


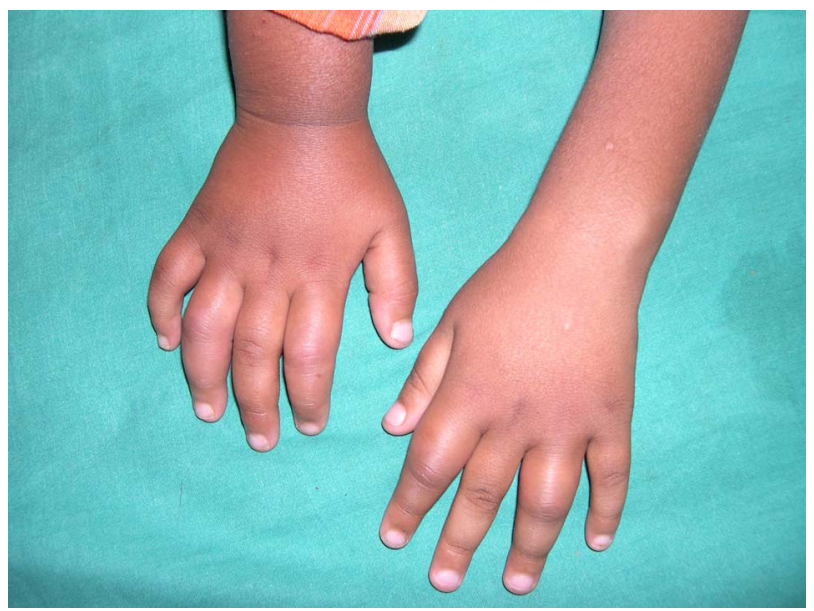

Figure 1. Hands of the child showing inflammed, swollen metacarpo-phalangeal and interphalangeal joints.

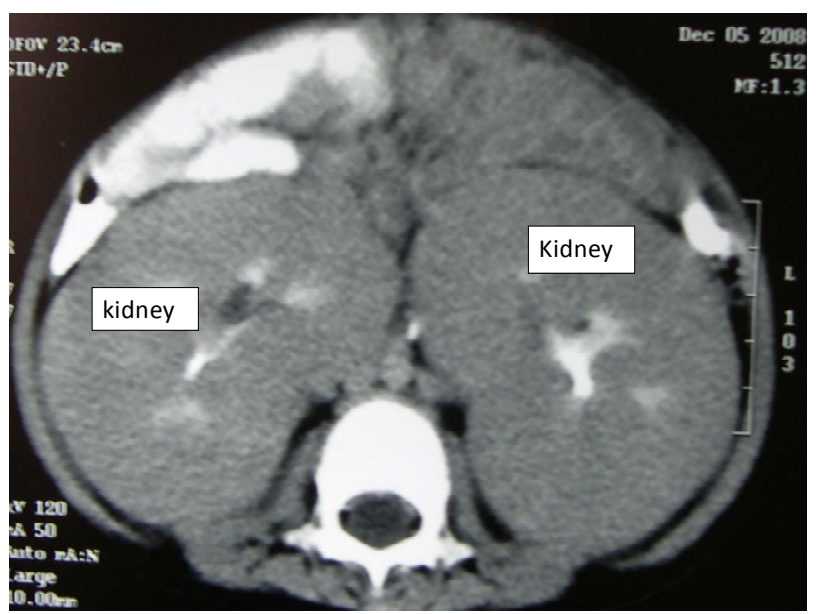

Figure 2. Contrast CT scan of abdomen showing grossly enlarged and diffuse bulky kidneys.

monoclonal light chains, $\mathrm{T}$ cell and myeloid markers were absent suggesting pre-B ALL. Cerebrospinal fluid examination did not reveal any evidence of meningeal infiltration. The final diagnosis was Pre-B acute lymphoblastic leukemia.

Hospital course: He was subjected to ALL BFM90 regime after confirmation of the diagnosis [1]. He received $12.5 \mathrm{mg}$ intrathecal methotrexate on Day 1, oral prednisolone (60 $\mathrm{mg} / \mathrm{m}^{2} /$ day) daily for 7 days and intravenous fluid during pre-induction phase. Thereafter phase 1 induction was started with intravenous vincristine (1.4 $\left.\mathrm{mg} / \mathrm{m}^{2}\right)$, doxorubicine $\left(30 \mathrm{mg} / \mathrm{m}^{2}\right)$, deep intramascular l-asparaginase $\left(10000 \mathrm{u} / \mathrm{m}^{2}\right)$, intrathecal methotrexate (12.5 mg) and oral prednisolone $\left(60 \mathrm{mg} / \mathrm{m}^{2}\right)$ according to the BFM90 protocol. We noticed dramatic response during the induction phase and the kidneys became non palpable within 10 days after starting chemotherapy. Repeat USG abdomen revealed normal size kidneys 4 wk later. Elevated uric acid level $(13.5 \mathrm{mg} / \mathrm{dl})$ was de- tected during his hospital stay. It was treated with adequate intravenous fluid, alkalization of urine and oral allopurinol. The joint symptoms subsided with normalization of uric acid level. The child had just completed the phase 1 induction chemotherapy at the time of reporting it and was doing well.

\section{DISCUSSION}

The unique combination of nephromegaly and transient intermittent arthritis of small joints in ALL has not been described in literature till date to the best of our knowledge. Children with ALL characteristically present with fever, pallor, bleeds, lymphadenopathy and organomegaly. Although renal infiltration is relatively frequent in acute lymphoblastic leukemia, clinically palpable renal enlargement occurs only in $2 \%-5 \%$ of cases. Isolated renal enlargement as a primary presenting feature of ALL is extremely rare [2-5]. Renal enlargement in a case of leukemia may be due to leukemic infiltration or simple hypertrophy or hyperplasia of cells [6]. Enlarged kidneys do not always indicate leukemic involvement as shown in a study by Frei et al. [6], where, as many as $30 \%$ of the kidneys in leukemic patients were free of malignant cells on autopsy. Therefore, kidney mass oriented approach for confirmation of diagnosis leaving aside bone marrow examination may lead to high possibility of missing the correct diagnosis. Nephromegaly without any leukemic infiltration is generally associated with heaptosplenomagaly [6]. The index patient presented only with nephromegaly. Renal functions are usually well preserved in such a situation as in the present case and only a few cases of renal failure secondary to a diffuse bilateral parenchymal infiltration are reported in the literature [7]. Although the role of radiotherapy in the treatment of leukemic infiltrates has been studied in the past, currently systemic chemotherapy remains the basic treatment even with leukemic infiltrates. Enlarged kidneys in ALL are thought to be an unfavourable sign. The index patient, however, responded well to chemotherapy and follow up ultrasound revealed normal size kidneys.

Joint involvement as an initial presentation of acute leukemia in children has been described in literature [8-11]. About $15 \%$ to $30 \%$ of ALL patients manifest with osteoarthitic symptoms, some of which may mimic juvenile idiopathic arthritis (JIA) [11]. However, the interesting fact was that the arthritis here was transient and intermittent. The exact mechanism of this transient arthritis was not clear. Although high uric acid, end product of leukocyte nucleic acid breakdown, causes arthritis it seems that it is not a causative agent here as it is normal to begin with the disease. A ten years survey in paediatric rheumatology clinic conducted by Trapani et al. [8] found 6 cases of ALL with arthritis out of 1254 patients. JIA was the most frequent provisional diagnosis and monoarticu- 
lar arthritis of large joint was the commonest mode of presentation. Investigators from Europe, North and South America have reported a similar pattern of joint involvement in ALL patients with osteoarthritic manifestation [9].

In another study by Jung et al. [10], out of 30 children initially diagnosed as JIA, 3 were later diagnosed as acute leukemia (2 ALL, 1 AML) by bone marrow examination. Among these 3 patients, one had monoarticular arthritis of large joint, another had arthritis in 3 large joints and third patient had only arthralgia. All had absence of blasts in peripheral smear which was similar to our case. Robazzi et al. [11] observed arthritis in 26\% occasions in a pool of 313 cases of ALL and it was mainly large joint arthritis. Marwaha et al. has reported an incidence of $16 \%$ of small joints involvement in a recently published article. There was no mention of transient intermittent arthritis [9]. The index case presented here with transient arthritis of small joints of hands and feet in contrast. The literature on comparison of children with ALL whose initial diagnosis was JIA, with other ALL patients, for the pattern of initial presentation, management, outcome, and prognostic factors is scant.

\section{CONCLUSION}

Bilateral enlargement of kidneys and arthritis in another normal child should raise the suspicion of acute leukemia and the patient should be subjected to bone marrow examination before ordering for other invasive tests.

\section{REFERENCES}

[1] Schrappe, M., Reiter, A., Ludwig, W.D., et al. (2000) Improved outcome in childhood acute lymphoblastic leukemia despite reduced use of anthracyclines and cranial radiotherapy: Results of trial ALL-BFM 90. Blood, 95, 3310-3322.

[2] Boueva, A. and Bouvier, R. (2005) B-cell lympholblastic leukemia as a cause of bilateral nephromegaly. Pedi- atric Nephrology, 20, 679-682. doi:10.1007/s00467-004-1740-5

[3] Basker, M., Scott, J.X., Ross, B., et al. (2002) Renal enlargement as primary presentation of acute lymphoblastic leukemia. Indian Journal of Cancer, 39, 154-156.

[4] Rudramurthy, P., Madhumathi, S.D., et al. (2008) Bilateral nephromegaly simulating wilms tumour: A rare initial manifestation of acute lymphoblastic leukemia. Journal of Pediatric Hematology/Oncology, 30, 471-473. doi:10.1097/MPH.0b013e318168e7b3

[5] Ali, S.H., Yacoub, F.M. and Al Matar, E. (2008) Acute lymphoblastic leukemia presenting as bilateral renal enlargement in a child. Medical Principles and Practice, 17, 504-506. doi:10.1159/000151576

[6] Frei III, E., Fritz, R.D., Price, E., et al. (1963) Renal and hepatic enlargement in acute leukemia. Cancer, 16, 10891092. doi:10.1002/1097-0142(196308)16:8<1089::AID-CNCR 2820160817>3.0.CO;2-1

[7] Glicklich, D., Sung, M.V. and Frey, M. (1986) Renal failure due to lymphomatous infiltration of the kidneys. Cancer, 58, 748-753. doi:10.1002/1097-0142(19860801)58:3<748::AID-CNC $\underline{\mathrm{R} 2820580323>3.0 . \mathrm{CO} ; 2-\mathrm{U}}$

[8] Trapani, S., Grisolia, F., Simonini, G., et al. (2000) Incidence of occult cancer in children presenting with musculoskeletal symptoms: A 10 yr survey in pediatric rheumatology unit. Semin Arthritis Rheum, 29, 348-359. doi:10.1053/sarh.2000.5752

[9] Marwaha, R.K., Kulkarni, K.P., Bansal, D. and Trehan, A. (2010) Acute lymphoblastic leukemia masquerading as juvenile rheumatoid arthritis: Diagnostic pitfall and association with survival. Annals of Hematology, 89, 249-254. doi:10.1007/s00277-009-0826-3

[10] Jung, A. and Nielsen, S.M. (1998) Arthritis as first symptom of leukemia in children. Ugeskrift for Laeger, $\mathbf{1 6 0}$, 2889-2890.

[11] Robazzi, T.C., Barreto, J.H., Silva, L.R., et al. (2007) Osteoarticular manifestations as initial presentation of acute leukemias in children and adolescents in Bahia, Brazil. Journal of Pediatric Hematology/Oncology, 29, 622-626. 